\title{
On the Impact of Amplitude Variation on Interpolated DFT Method and How to Reduce It
}

\author{
Wojciech Oliwa, Grzegorz Wieczorek \\ Institute of Electronics, Silesian University of Technology, \\ Ul. Akademicka 16, 44-100 Gliwice, Poland \\ woliwa@polsl.pl
}

\begin{abstract}
The Interpolated DFT is often used to improve frequency estimation of the spectral lines of DFT's spectrum. If the measured spectral line is distorted the accuracy of Interpolated DFT is decreased. Such distortion could be a result of amplitude variation during the acquisition of samples. To reduce the impact of amplitude changes a novel algorithm of digital amplitude stabilization was proposed. The simulations have been made to verify proposed algorithm. The amplitude variations were modelled by amplitude modulation of the measured signal. The relationship between systematic error and modulating frequency, phase, modulation index of an amplitude modulation and measured signal frequency were presented for two Interpolated DFT methods, with and without amplitude stabilization. Simulation tests prove that the application of amplitude stabilization (AS) algorithm significantly reduces the systematic error of the frequency estimation using both Interpolated DFT methods.
\end{abstract}

Index Terms-Discrete Fourier Transform; Amplitude stabilization; Frequency estimation; Frequency measurement; Interpolated DFT.

\section{INTRODUCTION}

The Discrete Fourier Transform is a very common and useful tool in signal analysis, as well as in various measurements, but it has a great disadvantage - low spectral resolution. Fortunately it can be improved using Interpolated DFT (IpDFT) methods, first introduced by Rife and Vincent in 1970 [1] and modified in many ways [2]-[6], [7].

Indeed, the IpDFT method is a simple and very accurate method of estimating signal parameters even if the measured signal is distorted or of low quality, but only if the spectral line of measured signal is not distorted. Such deformation is caused by two or more spectral lines overlapping and always leads to systematic error of the IpDFT method. Overlapping can be a result of the occurrence of signals with almost equal alias frequencies or as a result of signal modulation. In fact, the measured signal instability during the acquisition of the samples could be treated as amplitude or frequency modulation and modulation produces spectral lines around the measured signal's spectral line. If the modulating frequency is low enough these additional spectral lines cause distortion of the measured spectral line.

There is no known general method of reducing the impact

Manuscript received 28 September, 2017; accepted 21 January, 2018.

Publication supported by the Rector's grant in the field of scientific research and development works. Silesian University of Technology, grant number 02/030/RGJ18/0036 of signal variation (especially variation of the amplitude) on IpDFT methods. In special case, an exponentially decaying sinusoid, the IpDFT algorithm was proposed in [5] and [6]. The problem of amplitude changes was considered from a different point of view, namely, amplitude estimation in [8] and was presented in a general way in [9]-[11]. This paper presents a novel method to reduce the impact of amplitude variation on the frequency estimation using IpDFT methods in general case.

\section{USED MODEL AND METHODS}

As mentioned earlier, the variation of amplitude during samples acquisition could be modelled as an amplitude modulation (AM) and takes a form of

$$
\begin{aligned}
x(t)= & \left(A_{0}+A_{1} \times \cos (2 \pi \times f \times t+\phi)\right) \times \\
& \times \cos (2 \pi \times F \times t+\Phi)= \\
=A_{0} \times & (1+m \times \cos (2 \pi \times f \times t+\phi)) \times \\
& \times \cos (2 \pi \times F \times t+\Phi),
\end{aligned}
$$

where $A_{0}, F$ and $\Phi$ are the amplitude, the phase and the frequency of the measured signal, respectively $A_{l}, f$ and $\varphi$ are the amplitude, the phase and the frequency of distortion, respectively, and $m$ is the modulation index.

From many variants of the Interpolated DFT methods two were chosen: the one used in [11] and the one proposed in [6]. The first method was selected as this paper is in reference to [11] - this method will be called SIDFT (Simple Interpolated DFT). The second one was chosen due to the fact that it is an analytically derived solution for a damped sinusoid [6], and a damped sinusoid could be modeled with good accuracy by amplitude modulation [9] it will be called RVCI (Rife-Vincent Class I). Both methods are exact estimation of frequency if the signal is purely sinusoidal for the Hanning windowing function. Therefore only this window will be considered in detail, although some remarks on using other windows will be made too.

The SIDFT consists of two steps. Firstly, the auxiliary coefficient $\gamma\left(\delta_{p}\right)$ is calculated using the values of two greatest points on spectral line, $A(L)$ and $A(L-1)$ or $A(L+$ 1)

$$
\gamma\left(\delta_{p}\right)=\left\{\begin{array}{lll}
\frac{A(L)+2 \times A(L+1)}{A(L)+A(L+1)}, & \text { for } & A(L+1)>A(L-1), \\
\frac{A(L)+2 \times A(L-1)}{A(L)+A(L-1)}, & \text { for } & A(L+1)<A(L-1) .
\end{array}\right.
$$


Second, the frequency correction factor $\delta_{p}$ is determined

$$
\delta_{p}=\frac{1}{2} \times \frac{\gamma\left(\delta_{p}\right)-\gamma(0)}{\gamma(0.5)-\gamma(0)},
$$

where $\gamma(0)$ and $\gamma(0.5)$ are calculated using (2) and are constant for the chosen window.

The RVCI method is a little more complicated. First, using the amplitudes of the three greatest points two ratios, $R_{l}$ and $R_{2}$, are calculated:

$$
\left\{\begin{array}{l}
R_{1}=\frac{|A(L+1)|^{2}}{|A(L)|^{2}}, \\
R_{2}=\frac{|A(L-1)|^{2}}{|A(L)|^{2}},
\end{array}\right.
$$

and then the frequency correction factor $\delta_{p}$ is determined

$$
\delta_{p}=\frac{3}{2} \times \frac{R_{1}-R_{2}}{4 R_{2} \times R_{2}-R_{1}-R_{2}-2} .
$$

\section{THE SOURCES OF ERRORS}

Taking into account only the amplitude variation of measured signal, modelled by (1), the systematic error of the IpDFT depends on the following factors:

- the frequency $f$ and the phase $\varphi$ of the modulating signal, which will be called AM frequency and AM phase, respectively - this dependence is very irregular as it is shown in Fig. 1,

- the modulation index $m$ - only the ratio (not the absolute values) of both amplitudes $A_{0}$ and $A_{1}$ affect the spectrum, the modulation index role looks a little bit like an amplification factor of the influence of the modulation frequency and phase (Fig. 2),

- the fractional part of the signal frequency expressed in bins, i.e. the value of frequency correction calculated by IpDFT (3) or (5) - this has almost periodical dependency (with period equal to $1 \mathrm{bin}$ ),

- the integer part of the signal frequency expressed in bins, i.e. the index of the discrete spectrum bin with the greatest amplitude - this has a negligible influence, except in cases where the measured frequency is near the ends of the spectrum. This occurs due to a spectral leakage from the "negative" frequency spectral line [7].

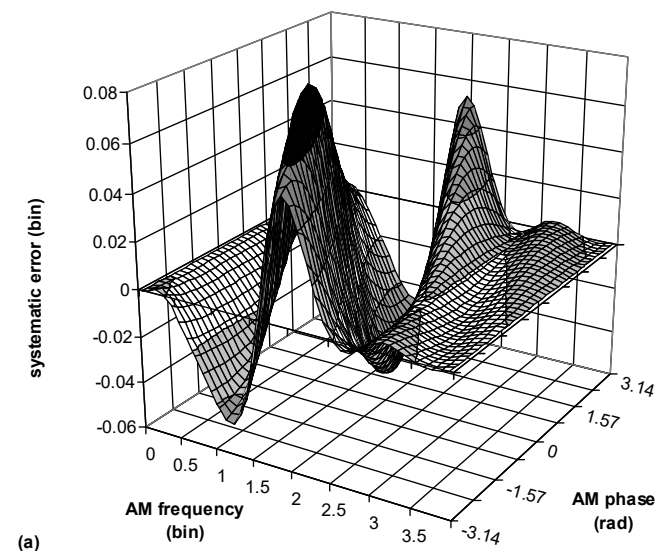

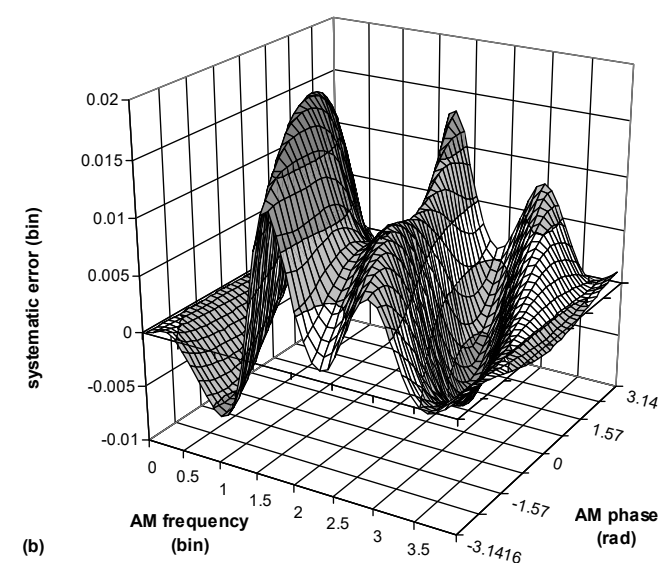

Fig. 1. An example of systematic error vs. AM frequency and AM phase dependence. These relationships are almost independent of the integer part of signal frequency expressed in bins. The modulation index is 0.4 , FFT size 1024, signal frequency in bins 217.3. (a) SIDFT (b) RVCI.

As always the additive noise is a source of frequency estimation uncertainty and the signal-to-noise ratio (SNR) usually expresses noise influence on signal.
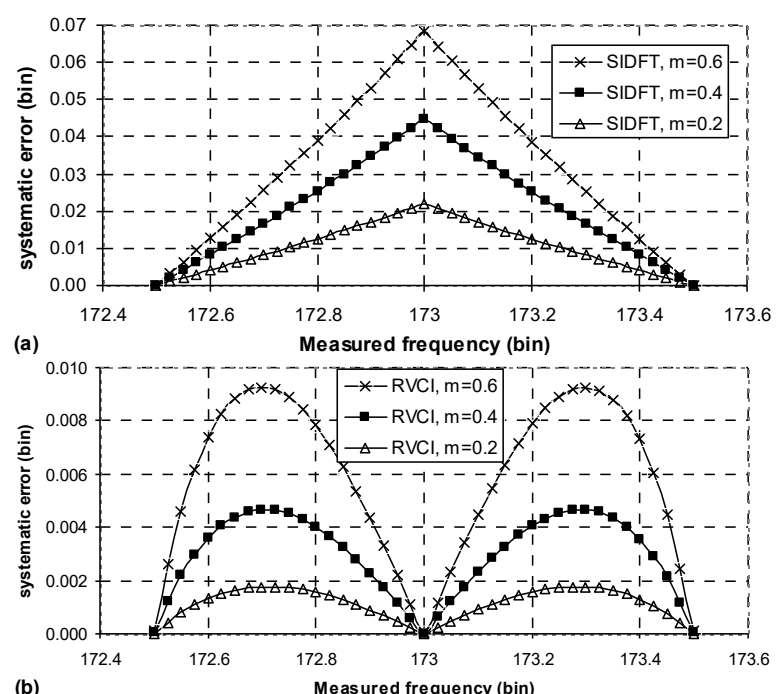

ig. 2. The dependence of mean absolute systematic error vs. fractional signal frequency. The modulation factor behaves like the amplification factor. Each point is a mean of absolute values of systematic errors for 1600 different combinations of AM frequency (from a range in bins 0 to 3.9) and AM phase (from a range $-\pi$ to $\pi$ ). FFT size 1024. (a) SIDFT (b) RVCI.

\section{Digital Amplitude Stabilization}

It is a quite complicated task to analytically solve the problem of the influence of amplitude modulation on the IpDFT as it should use at least 5 bins since there are 4 factors to calculate (the signal frequency, the modulation index, the amplitude modulation phase and frequency). Even if an analytical solution was derived it would not completely solve the problem of random amplitude variation. A different way to reduce the impact of the amplitude changes is to stabilize it after sampling (in a digital domain). This is a more general approach and could be applied to the signal with a random amplitude variation.

The following amplitude stabilization algorithm (AS), that corrects the amplitude of the acquired samples, was developed (Fig. 3):

1. Calculate the mean value of the samples.

2. Find the mean-crossing points $x_{b}(k)$ and $x_{e}(k)$.

3 . Find the peak value $A_{p}(k)$ between points $x_{b}(k)$ and $x_{e}(k)$. 
4. Use the reciprocal of $A_{p}(k)$ as an amplitude correction factor and multiply all samples between points $x_{b}(k)$ and $x_{e}(k)$ by that factor.

5. Use point $x_{e}(k)$ as new $x_{b}(k)$, increment $k$ and repeat steps (2)-(4) for all remaining samples.

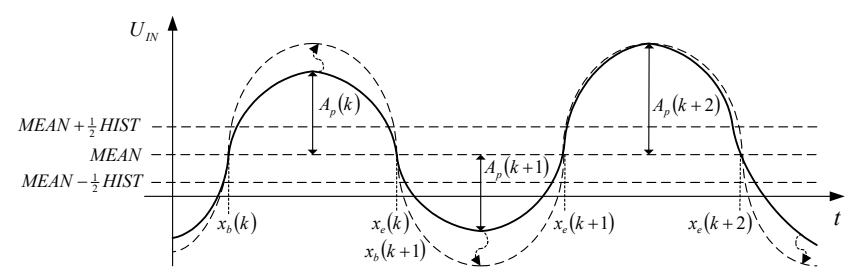

Fig. 3. The idea of digital amplitude stabilization.

The hysteresis is introduced to decrease sensitivity to noise and distortion, but is not necessary for a good quality signal.

\section{ANALYSis OF Simulation RESUlts}

As mentioned above, in the case of the amplitude modulated signal there are many factors that influence a systematic error of the IpDFT. The simulation tests seem to be the simplest way to control all of these factors. Fortunately the actual properties of the meter using the IpDFT are consistent with simulation and analytical predictions [11].

TABLE I. THE PROPERTIES OF INTERPOLATED DFT METHODS AFTER AMPLITUDE STABILIZATION. MODULATION INDEX 0.4.

\begin{tabular}{|c|c|c|c|c|}
\hline Parameter & $\begin{array}{c}\text { SIDF } \\
\text { T }\end{array}$ & RVCI & $\begin{array}{c}\text { SIDFT } \\
+ \text { AS }\end{array}$ & $\begin{array}{c}\text { RVCI + } \\
\text { AS }\end{array}$ \\
\hline $\begin{array}{c}\text { Mean of absolute values of } \\
\text { errors }\end{array}$ & 0.0214 & 0.0084 & $\mathbf{0 . 0 0 0 5}$ & $\mathbf{0 . 0 0 0 5}$ \\
\hline Maximum absolute error & 0.0447 & 0.0191 & 0.0355 & 0.0326 \\
\hline Rms error & 0.0258 & 0.0104 & 0.0017 & 0.0015 \\
\hline $\begin{array}{c}\text { Probability of absolute } \\
\text { error less than 0.001 (bin) }\end{array}$ & $24.8 \%$ & $44.6 \%$ & $90.74 \%$ & $90.57 \%$ \\
\hline $\begin{array}{c}\text { Probability of absolute } \\
\text { error less than 0.005 (bin) }\end{array}$ & $46.2 \%$ & $78.0 \%$ & $98.13 \%$ & $98.17 \%$ \\
\hline $\begin{array}{c}\text { Probability of absolute } \\
\text { error less than 0.01 (bin) }\end{array}$ & $57.3 \%$ & $94.5 \%$ & $99.32 \%$ & $99.32 \%$ \\
\hline
\end{tabular}

Simulations show that the application of the AS algorithm significantly reduces the expected systematic error of both IpDFT methods, however the maximum error for RVCI increases (Table I) and there are few signal frequencies with noticeably greater error (Fig. 4). Using AS has a side effect as it produces harmonics of the measured signal. In rare cases the spectral lines of harmonics deform the measured signal's spectral line (what we have tried to avoid happens in this case). The worst case occurs when the signal frequency expressed in bin has a value of one third of the DFT size (Fig. 4). However this overlapping appears rather rarely and the AS algorithm noticeably decreases the mean absolute error (i.e. the expected error) and increases the probability of obtaining an accurate estimation of measured frequency (Table I). The probability calculations in Table I were based on 8 million different simulations (5000 different signal frequencies multiplied by 1600 different combinations of amplitude modulation frequencies and phases) and take into account only systematic error.

Figure 5 presents an exemplary relationship between the mean absolute systematic error of the IpDFT methods (and the AS algorithm combined) and the frequency correction factor and the modulation index for the case without spectral lines overlapping. One should notice that Fig. 2 shows the same error in the same conditions without using the AS algorithm. Comparison of Fig. 4 and Fig. 2 shows that the AS algorithm reduces the systematic error of the frequency estimation by a factor of about 150 for SIDTF and by a factor of about 22 for RVCI.

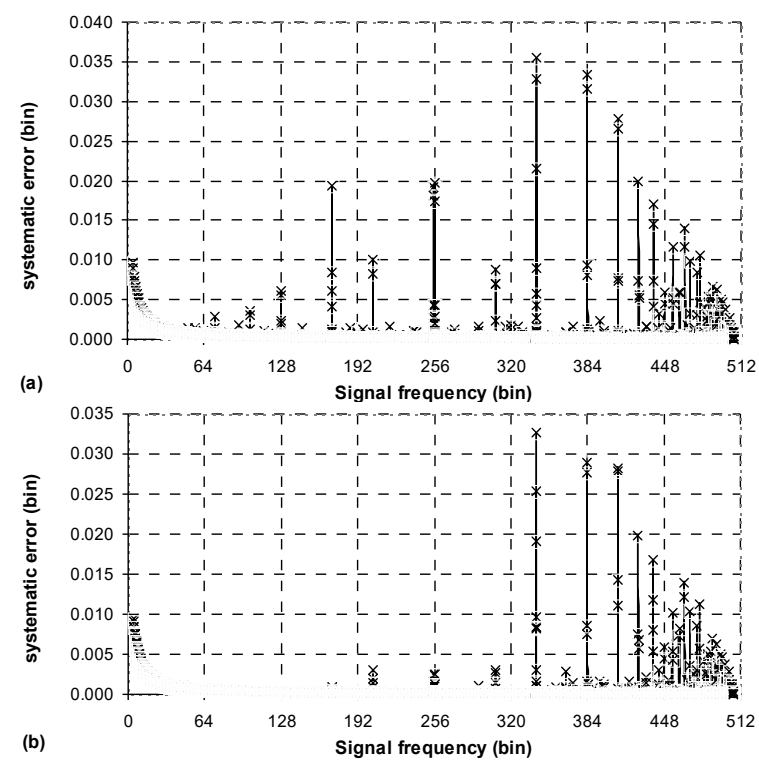

Fig. 4. The mean absolute systematic error vs. signal frequency. Signal frequency changes (in bins) in range from 5 to 506 with step 0.1. Each point is a mean of absolute values of systematic errors for 1600 different combinations of AM frequency (from a range in bins 0 to 3.9) and AM phase (from a range $-\pi$ to $\pi$ ). FFT size 1024 . (a) SIDFT+AS (b) RVCI+AS.
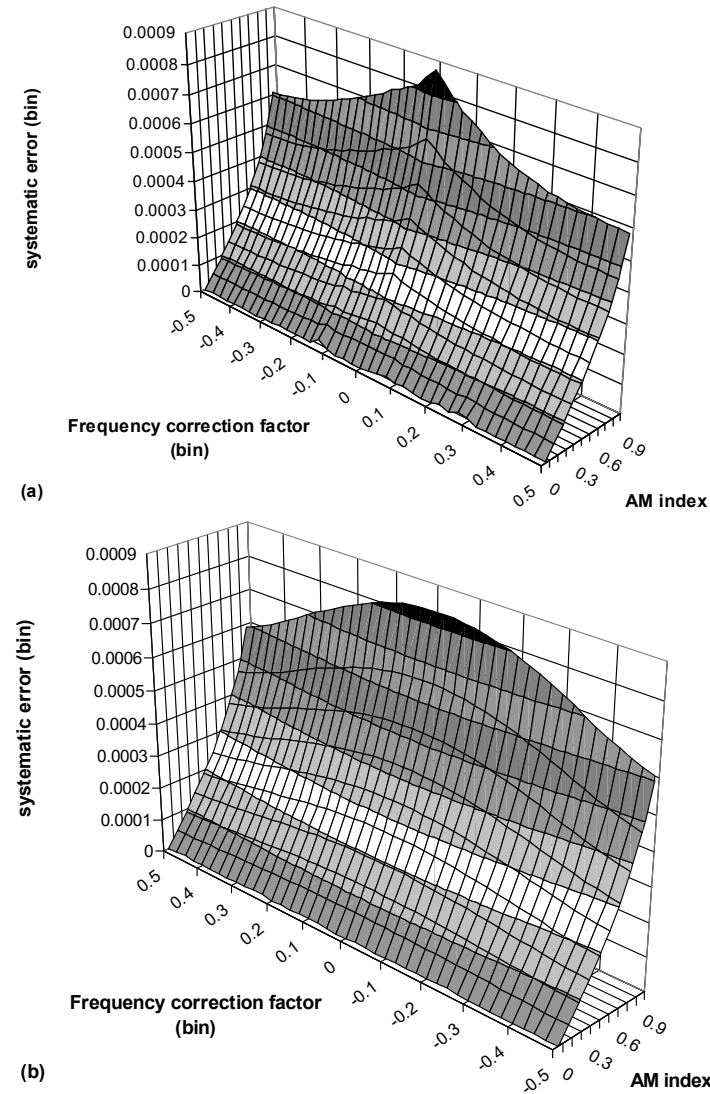

Fig. 5. An exemplary dependence of mean absolute systematic error vs fractional signal frequency and modulation index. Each point is a mean of absolute values of systematic errors for 1600 different combinations of AM frequency (from a range in bins 0 to 3.9) and AM phase (from a range $-\pi$ to $\pi$ ). Signal frequency (in bins) $172.5-173.5$, FFT size 1024. (a) SIDFT+AS (b) RVCI+AS. 
The influence of random noise on estimation of uncertainty was also verified by adding Gaussian noise to the measured signal and repeating every simulation 1000 times. The standard deviation dependences on SNR compared to SIDFT's standard deviation were presented in Fig. 6. As was expected using the AS algorithm increases the standard deviation, but it is a small change of only about $8 \%$ for both IpDFT methods.

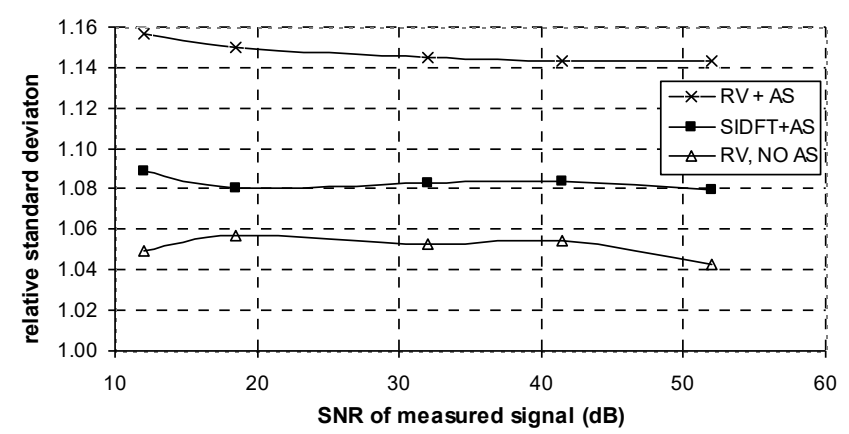

Fig. 6. A standard deviation relative to the SIDFT's (without AS) standard deviation. Each point is a mean of 1000 simulations for 1600 different combinations of AM frequency (from a range in bins 0 to 3.9) and AM phase (from a range $-\pi$ to $\pi$ ). Signal frequency 217.3 , modulation index 0.4 , FFT size 1024.

\section{DISCUSSION}

It should be mentioned that the proposed AS algorithm could be used when there is one dominant spectral line in the spectrum and the amplitude of the signal is unstable. In this case, the estimation of frequency using IpDPT methods can be done with high accuracy and little computational complexity. If there are two or more spectral lines with similar amplitudes the filtering is needed before application of AS.

The SIDFT is notably more sensitive than RVCI to the measured signal's amplitude changes. However, both IpDFT methods have almost equal accuracy if the AS algorithm is used. Moreover, given the general similarity of the different IpDFT methods it is reasonable to expect the AS algorithm to be useful without having tested other IpDFT methods.

Although only the Hanning windowing function was used, the SIDFT method is an universal method for most common windowing functions. Other tests that were conducted confirmed that the AS algorithm also improves frequency estimation with Kaiser-Bessel, Dolph-Chebyshev and Blackmann-Harris windows.

The tests in which the amplitude of measured signal changed randomly (although with a limitation, i.e. the amplitude was stable during the time longer that the period of measured signal) were also conducted. The results of these tests strongly suggest that proposed AS algorithm is efficient in general cases.

\section{CONCLUSIONS}

The simple method of reducing the sensitivity of frequency estimation using the IpDFT on amplitude variations during the acquisition of samples was introduced and verified by simulation tests. This method performs amplitude stabilization before calculating the DFT and IpDFT. For almost all cases the AS significantly decreases the systematic error of IpDFT methods, although in rare situations it increases this error.

\section{REFERENCES}

[1] D. C. Rife, G. A. Vincent, "Use of the discrete Fourier transform in the measurement of frequencies and levels of tones", Bell System Technical Journal, vol. 49, no. 2, pp. 197-228, 1970. DOI: 10.1002/j.1538-7305.1970.tb01766.x.

[2] W. Oliwa, "An improved approach to interpolated DFT", PDS 2001 Gliwice 2001

[3] L. Fan, G. Qi, "A new synthetic frequency estimation method of sinusoid based on interpolated FFT", Fifth Int. Conf. Instrumentation and Measurement, Computer, Communication and Control (IMCCC 2015), 2015. DOI: 10.1109/IMCCC.2015.366.

[4] K. Duda, S. Barczentewicz, "Interpolated DFT for $\sin ^{\alpha}(\mathrm{x})$ windows", IEEE Trans. Instrumentation and Measurement, vol. 63, no. 4, pp. 754-760, 2014. DOI: 10.1109/TIM.2013.2285795.

[5] R-Ch. Wu, Ch-T. Chiang, "Analysis of the exponential signal by the interpolated DFT algorithm", IEEE Trans. Instrumentation and Measurement, vol. 59, no. 12, pp. 3306-3317, 2010 DOI: 10.1109/TIM.2010.2047301.

[6] K. Duda, L. B. Magalas, M. Majewski, T. P. Zielinski, "DFT-based estimation of damped oscillation parameters in low-frequency mechanical spectroscopy", IEEE Trans. Instrumentation and Measurement, vol. 60, no. 11, pp. 3608-3618, 2011. DOI: 10.1109/TIM.2011.2113124.

[7] Y. Sun, C. Zhuang, Z. Xiong, "A scale factor-based interpolated DFT for chatter frequency estimation", IEEE Trans. Instrumentation and Measurement, vol. 64, no. 10, pp. 2666-2678, 2015. DOI: 10.1109/TIM.2015.2421711.

[8] J. Borkowski, D. Kania, "Interpolated-DFT-based fast and accurate amplitude and phase estimation for the control of power", Metrology and Measurement Systems, vol. 23, no. 1, pp. 13-26, 2016. DOI: 10.1515/mms-2016-0013

[9] W. Oliwa, "An influence of signal amplitude variation on interpolated DFT method", in IEEE Conf. Publication Int. Conf. Signals and Electronic Systems (ICSES 2010), 2010, pp. 77-80. [Online]. Available: http://ieeexplore.ieee.org/document/5595248

[10] D. Agrez, "Estimation of the instantaneous periodic parameters in the frequency domain", Instrumentation and Measurement Technology Conf. (IMTC 2006), 2006, pp. 1731-1735. DOI: 10.1109/IMTC.2006.328221.

[11] W. Oliwa, G. Wieczorek, "Fast and accurate frequency meter using the interpolated DFT method", Elektonika ir elektrotechnika, vol. 23, no. 23, pp. 43-46, 2017. DOI: 10.5755/j01.eie.23.3.18331. 